\title{
Identifying Lagrangian Coherent Vortices in a Mesoscale Ocean Model
}

\author{
Nathaniel Tarshish ${ }^{\mathrm{a}}$, Ryan Abernathey ${ }^{\mathrm{b}, \mathrm{c}}$, Ci Zhang ${ }^{\mathrm{b}, \mathrm{c}}$, Carolina O. Dufour ${ }^{\mathrm{d}}$, \\ Ivy Frenger ${ }^{\mathrm{e}}$, Stephen M. Griffies ${ }^{\mathrm{a}, \mathrm{f}}$ \\ a Atmospheric and Oceanic Sciences Program, Princeton University, Princeton, New \\ Jersey, USA \\ ${ }^{b}$ Division of Ocean and Climate Physics, Lamont-Doherty Earth Observatory, USA \\ ${ }^{c}$ Department of Earth and Environmental Sciences, Columbia University in the City of New \\ York, USA \\ ${ }^{d}$ Department of Atmospheric and Oceanic Sciences, McGill University, Montreal, Quebec, \\ Canada \\ ${ }^{e}$ GEOMAR Helmholtz Centre for Ocean Research Kiel, Kiel, Germany \\ ${ }^{f}$ NOAA/Geophysical Fluid Dynamics Laboratory, Princeton, New Jersey
}

\begin{abstract}
We identify Lagrangian coherent vortices in a global mesoscale eddy-permitting ocean model using the rotation-based method of Haller et al. (2016). We present an analysis of the acute sensitivity of the identification results to varying the method's free parameters, and develop physically justified parameter choices that allow for systematic vortex identification. In contrast to prior vortex studies, we probe the broad spectrum of coherency in the ocean by determining free parameter choices that partition the spectrum into distinct coherency classes, allowing for the identification of strictly coherent, moderately coherent, and leaky vortices. Our tuning methodology is grounded in a combination of sensitivity analysis, convergence tests, and consideration of the ocean model's physics. To aid in this process, we introduce the Coherency Index, a novel Lagrangian diagnostic for mathematically quantifying the degree of material coherency of a Lagrangian vortex. We aim for this manuscript and the accompanying openaccess code to serve as a manual and toolset for the oceanographer interested in harnessing a rigorous Lagrangian method to uncover coherent structures in ocean models and observations.
\end{abstract}

Keywords: Coherent Structures, Mesoscale Eddies, Vortex Identification, Lagrangian Analysis, RCLV

Preprint submitted to Ocean Modeling

July 4, 2018 


\section{Introduction}

Transient ocean mesoscale fluctuations play a central role in the global climate system, transporting climate-relevant tracers such as heat and carbon. In satellite observations and numerical simulations, a portion of these fluctuations take the form of materially coherent vortices (i.e., vortices whose interior material coheres together). Consensus has not emerged, however, as to the abundance of these coherent vortices in the global oceans and the magnitude of the associated transport.

In the literature, coherent transport questions have been investigated primarily through the lens of Eulerian-based vortex identification: a set of techniques that attempt to diagnose coherent structures via anomaly contours of an Eulerian field (e.g., sea level anomaly or Okubo-Weiss parameter of Okubo (1970); Weiss (1991)). To compute transport estimates, these studies make the fundamental assumption that Eulerian "vortex contours" trap and transport their material interior (for recent examples, see Raj et al. (2016); Dong et al. (2014); Zhang et al. (2014a b)). Many studies justify this assumption by considering $U_{g} / c$, where $U_{g}$ is the maximum geostrophic speed within the vortex and $c$ is the mean translation speed (Chelton et al. 2007, 2011). When this diagnostic parameter is greater than unity, rotation dominates over translation, indicating that the identified feature departs from linear dynamics and "can advect a parcel of trapped fluid" (Chelton et al. 2011). As practitioners of this methodology acknowledge, the mere presence of some ability to trap fluid does not quantitatively guarantee complete material coherence over a vortex's total lifetime (Zhang et al., 2014a, dOvidio et al., 2013). Nonetheless, past studies make this assumption to reach estimates suggesting that identified vortices collectively play a significant role in global ocean transport (Dong et al., 2014. Zhang et al., 2014b Petersen et al. 2013).

On the other hand, several studies have called into question the ability of Eulerian vortex identification methods to correctly diagnose material transport 
(Abernathey \& Haller (2018); Haller et al. (2016); Beron-Vera et al. (2015); dOvidio et al. (2013); Froyland et al. (2007) and references therein). The community's development of rigorous Lagrangian-based methods has cast doubt on Eulerian vortex methodology, challenging the notion that Eulerian anomaly fields and diagnostics such as $U_{g} / c$ can reliably uncover material transport. 35 In contrast, Lagrangian techniques exploit material trajectories-the fundamental elements of fluid transport-and thus provide direct access to the transport structure of ocean flows.

A feature common to both Lagrangian and Eulerian vortex identification methods, however, is the prominent role of free parameters, including those related to numerical implementation. For a Lagrangian method, the number of free parameters varies from 3-4 (depending on the implementation) in the case of the Lagrangian-Averaged Vorticity Deviation (LAVD) method of Haller et al. (2016) and up to 6-7 for the geodesic "black hole vortex" method of Haller \& Beron-Vera (2013). An informative discussion of the strengths, weaknesses, and

45 free parameters of Lagrangian vortex algorithms can be found in Hadjighasem et al. (2017), which demonstrates promising results for the LAVD method. For reference, the identification of the popular Eulerian sea surface height (SSH) eddies of Chelton et al. (2011) required nine free parameters . $^{1}$

It may be inevitable that these free parameters exist. After all, a vortex identification algorithm must take a global field (e.g., sea surface height, velocity, LAVD) as input and return a discrete object as output. As in mathematics, passing from the continuous to the discrete limit requires the introduction of a threshold or tolerance.

\footnotetext{
${ }^{1}$ The free parameters of Chelton et al. (2011) are as follows: minimum SSH threshold for all pixels inside anti-cyclonic eddies, maximum SSH threshold for all pixels inside anti-cyclonic eddies, minimum SSH threshold for all pixels inside cyclonic eddies, maximum SSH threshold for all pixels inside cyclonic eddies, minimum number of pixels within eddy, maximum number of pixels within eddy, eddy SSH amplitude threshold, maximum distance between interior eddy points, and the choice of the number of pixels within a local neighborhood of an SSH extremum.
} 
The presence of free parameters presents a challenge for the oceanographer, whose desire is to identify physical structures that exist in the ocean independent of the particulars of an identification method. To complicate matters, the relationship between the mathematical parameters of the Lagrangian method and the physics of the identified structures is opaque. For example, distinguishing between coherent versus incoherent sets of trajectories with the transport operator or spectral clustering approach boils down to a delicate mathematical task: identifying a gap in the eigenvalues of an operator's spectrum (Froyland et al., 2007; Hadjighasem et al., 2016). Without exploring and understanding the physical significance of varying this parameter, the oceanographer cannot harness the method to objectively shed light on ocean transport.

65 In the applied mathematics literature that introduced these Lagrangian techniques, the methods' sensitivity and free parameters are not always systematically examined. For example, the authors of several leading vortex methods in the comparison paper of Hadjighasem et al. (2017) omit details on how the method was tuned: "We therefore rely on our expertise and experience to choose 70 a reasonable set of parameters for each method with the intention that (i) The choice of parameter(s) results in the most favorable outcome for the corresponding method and (ii) The outcome is robust, i.e., small variations in the parameters do not lead to drastic changes in the outcome". Thus, while the applied mathematics literature provides promising tools, it lacks instructions on how 75 to physically rationalize a tuning of the sensitive parameters. Furthermore, the literature lacks a clear definition of a "favorable outcome", which suggests that the final results are determined by the author's preferences rather reproducible objectives.

Our paper aims to help fill this gap in the existing literature by creating so a bridge that spans from the methods papers of the applied math community to the vortex census studies of oceanography. Our objective is to implement and apply a Lagrangian-based vortex identification method to a high-resolution global ocean model, conduct a rigorous and thorough sensitivity analysis, and arrive at a physically justified tuning that systematically identifies materially 
coherent vortices. We focus on a particular vortex identification method (the LAVD method of Haller et al. (2016)), but, in conducting our analysis, aim to provide a tuning methodology that is relevant for other techniques. Wherever possible, we strive to lend physical interpretation to the variation of the mathematical parameters. In doing so, we have taken steps to ensure that the tuning 90 is robust, in the sense that small adjustments to the free parameters do not produce large changes in the results.

Stemming from this effort, we introduce a new Lagrangian-based diagnostic of material coherency, which we refer to as the Coherency Index. This diagnostic allows us to precisely quantify the sensitivity of the identified vortices' coherency to varying the method's free parameters. Beyond this usage, the diagnostic has broad applicability, and could be employed to compare the coherency of vortices across data sets, models, and identification methods.

\section{Method and Materials}

\subsection{The LAVD Method}

Haller et al. (2016) introduced a Lagrangian-based vortex identification (abbreviated as the LAVD method) that exploits rotational coherence as a proxy for material coherence. We have implemented the LAVD identification method in an open-source Python package floater (available at https://github.com/rabernat/floater). The intuition for the method springs from the observation that fluid trajectories inside a coherent vortex are spatially and rotationally organized in bands, layered concentrically about a rotating near-circular core. This combined spatial and rotational organization makes it possible to detect materially coherent objects via a trajectory-based metric of rotation.

In particular, we consider two dimensional flow and examine the relative vorticity defined as $\zeta=\partial_{x} v-\partial_{y} u$ and equal to twice the fluid's local rotation speed relative to the Earth's rotation. Let a fluid element be labeled by a material label $\boldsymbol{a}$, and its position in physical space at time $t$ be notated as $\boldsymbol{X}(\boldsymbol{a}, t)$. We introduce the Lagrangian relative vorticity field, $\zeta(\boldsymbol{X}(\boldsymbol{a}, t) ; t)$, which takes 
as input a particular material trajectory and a specific time. For convenience, we choose to label the material by its position in physical space at some reference time $t_{0}$, so that $\boldsymbol{a}=\boldsymbol{x}_{0}$. We are interested in characterizing not just the instantaneous local rotation, but the average local rotation experienced by a material element over a finite time interval $\left[t_{\mathrm{i}}, t_{\mathrm{f}}\right]$. To do so, we define the Lagrangian-Averaged Vorticity Deviation,

$$
\operatorname{LAVD}_{t_{\mathrm{i}}}^{t_{\mathrm{f}}}\left(\boldsymbol{x}_{0}\right)=\frac{1}{t_{\mathrm{f}}-t_{\mathrm{i}}} \int_{t_{\mathrm{i}}}^{t_{\mathrm{f}}}\left|\zeta\left(\boldsymbol{X}\left(\boldsymbol{x}_{0}, \tau\right) ; \tau\right)-\overline{\zeta(\tau)}\right| d \tau
$$

where $\bar{\zeta}$ is the domain average of the relative vorticity field (Haller et al. 2016$)$. For a given time interval, LAVD assigns a single scalar to each trajectory, the magnitude of which describes the average amount of local rotation experienced by the material element. To understand the rotational structure of the flow, the convention is to visualize the Lagrangian field, $\operatorname{LAVD}_{t_{\mathrm{i}}}^{t_{\mathrm{f}}}\left(\boldsymbol{x}_{0}\right)$, by plotting the value for each material element in the element's initial physical location (i.e., $\left.\boldsymbol{X}\left(\boldsymbol{x}_{0}, t_{\mathrm{i}}\right)\right)$. An example visualization is shown in Figure 1 for the Kuroshio current in the NOAA/GFDL CM2.6 model (detailed in the following section). The algorithm utilizes this representation of $\operatorname{LAVD}_{t_{\mathrm{i}}}^{t_{\mathrm{f}}}\left(\boldsymbol{x}_{0}\right)$, which we simply refer to as "the LAVD field for $\left[t_{\mathrm{i}}, t_{\mathrm{f}}\right]$ ", to identify rotationally coherent vortices.

We now return to our earlier observation that coherent vortices are composed of collectively rotating fluid that is organized into concentric bands around a rotating near-circular core. Interpreting this feature in terms of the LAVD field provides the definition of a rotationally coherent Lagrangian vortex (RCLV) as a nested family of iso-LAVD contours containing an innermost LAVD maximum. The outer boundary of the RCLV is determined by a threshold on the convexity 125 of the contour ${ }^{2}$ A sample nested family of LAVD contours is shown for an Agulhas ring in Figure 2, As explored in detail in Section 3.3, the convexity threshold is the primary free parameter of the LAVD method. The coherency

\footnotetext{
${ }^{2} \mathrm{~A}$ contour is convex if the line segment joining any pair of interior points lies entirely within the contour (e.g., squares, ovals, and circles are convex, but crescents or star-shaped polygons are not).
} 


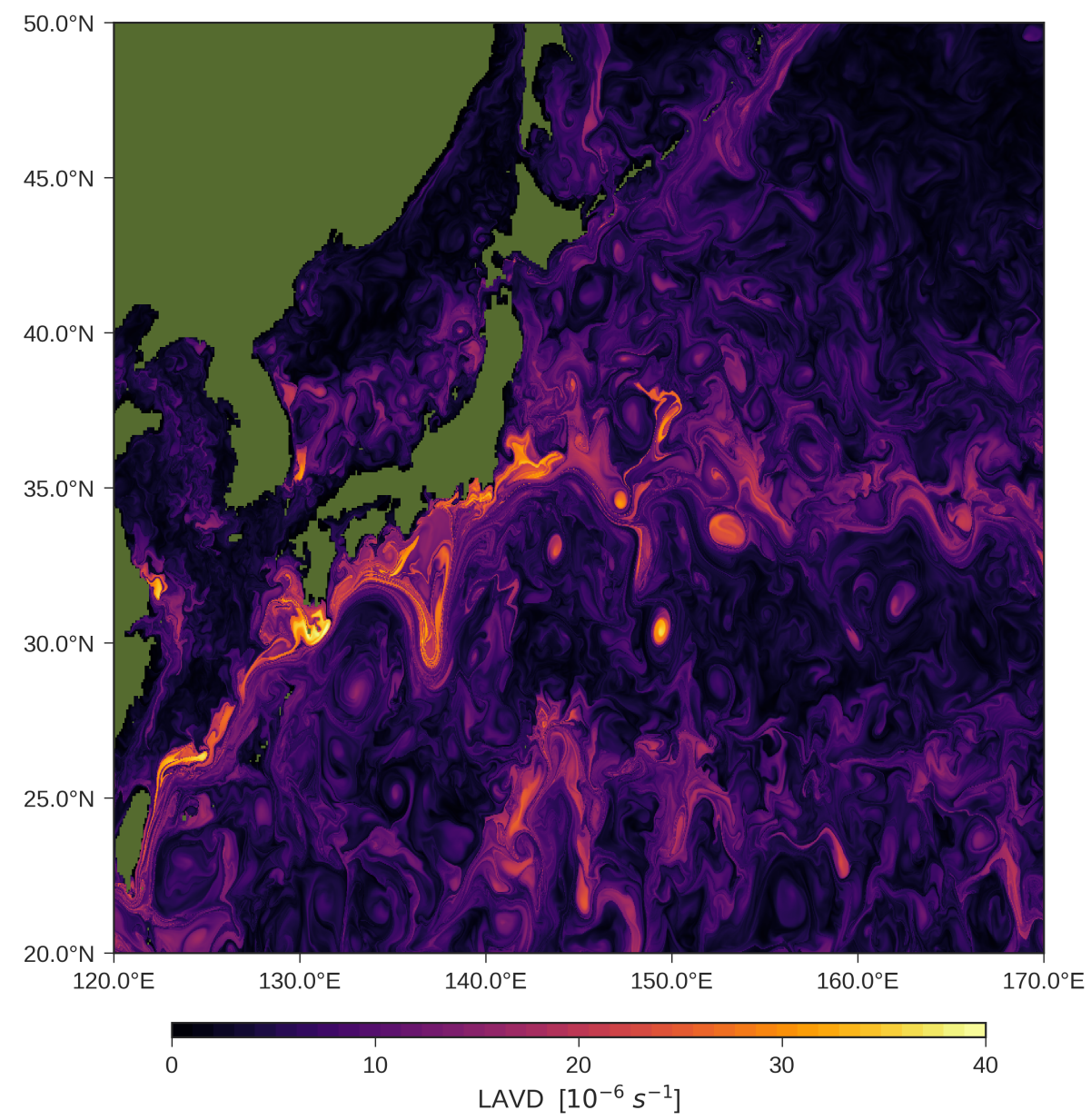

Figure 1: LAVD field computed over a 30-day interval in the Kuroshio region of the Northeast tropical Pacific Ocean. Smooth near-circular regions of LAVD anomaly represent mesoscale regions that rotate coherently over the subsequent 30-day interval.

and population of the identified RCLVs are acutely sensitive to the convexity threshold. The above presentation is oriented towards the oceanographer, but 130 we refer readers to Haller et al. (2016) and Haller (2016) for a rigorous and mathematical presentation of how LAVD naturally arises from a dynamic polar decomposition of the deformation gradient.

The definition of an RCLV relies on the empirically-supported hypothesis 


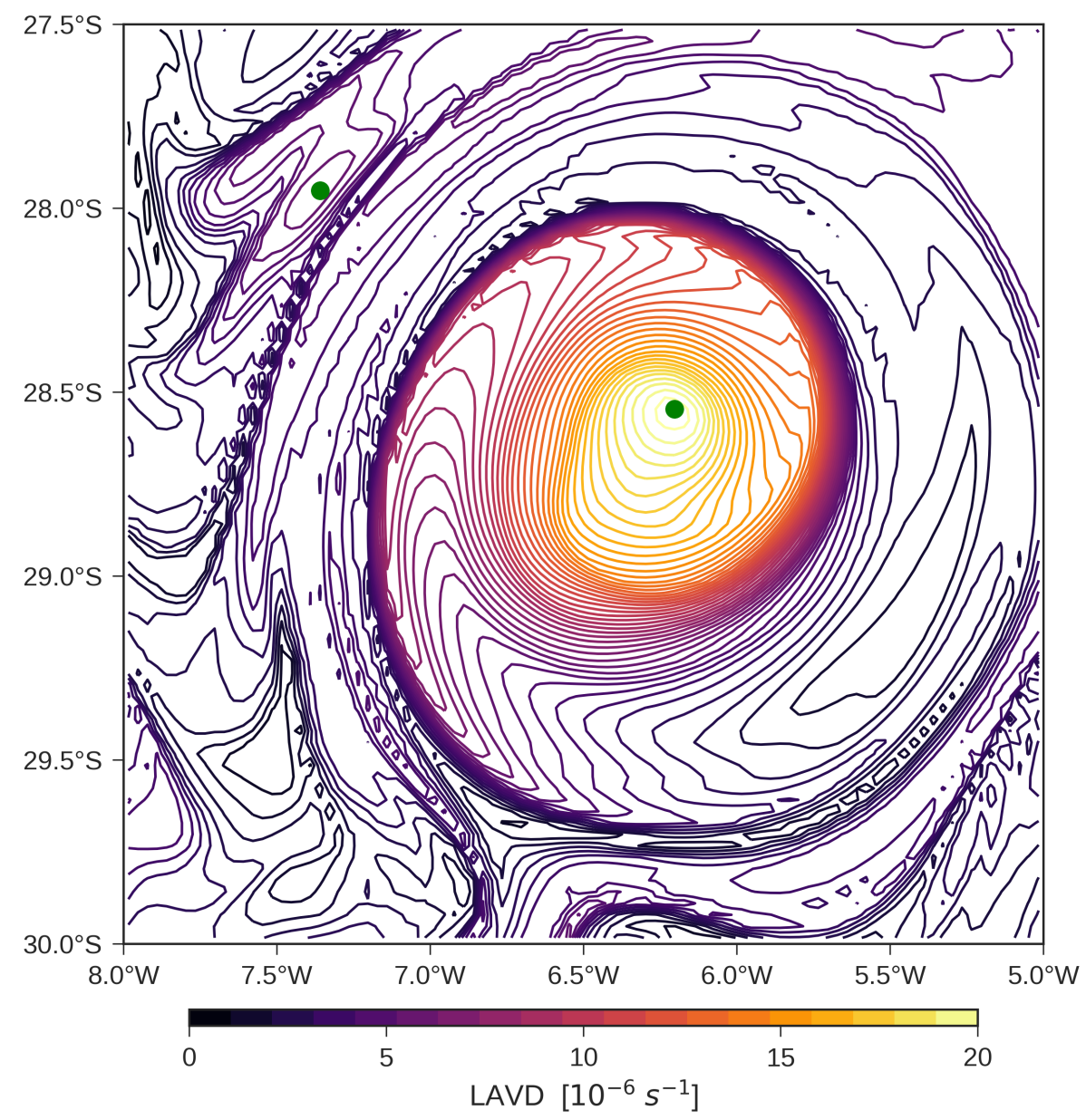

Figure 2: The contour field (shown in purple to yellow) of the Agulhas ring of Figure 8 identified with a contour increment of $4 \times 10^{-7} \mathrm{~s}^{-1}$. Local maxima are shown as green points. In practice, we use a contour increment of $10^{-8} \mathrm{~s}^{-1}$ for RCLV identification, but the resultant density of the contour field is so high that visualizations make the field look nearly continuous and are less pedagogical. 
that the more convex an LAVD contour, the more the interior fluid rotates together, and by extension, the more the fluid coheres. It is worth noting that this connection between LAVD contour convexity and material coherency, while critical to the method, lacks rigorous mathematical justification. For general finite contours, the justification is empirical and "motivated by the near-circular cross-section generally observed for stable vortices" Haller et al. (2016). The majority of Section 3.3 is devoted to investigating this empirical relationship.

As a preliminary demonstration of the method, we compare the evolution of vortex material identified by the method to randomly-selected "control" fluid. As shown in Figures 3 , the rotationally coherent object diagnosed by the method exhibits a high degree of material coherence. In this particular case, we find a downwelling vortex that contracts the surface material into a localized patch. (In general, we also find upwelling RCLVs that disperse material and non-divergent RCLVs that preserve their area). The control material, however, is widely dispersed and stirred with the environmental fluid.

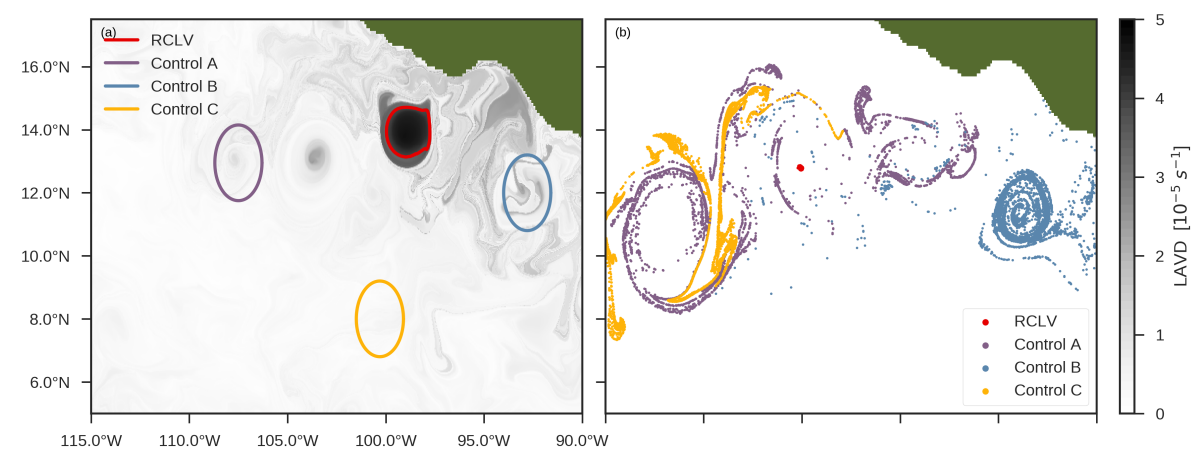

Figure 3: a) An LAVD field computed over a 60-day interval in the North Pacific Ocean. Contours indicate the detected RCLV boundary (shown in red) and randomly selected control volumes (shown in purple, blue, and gold). b) The material interiors after 60 days of advection by the surface flow (numerical details on particle advection are described in the subsequent section). The RCLV material (shown in red) contracts to a localized patch, a signature of a strongly downwelling vortex. 


\subsubsection{Implementation Details} identifying coherent vortices to the computational task of finding convex contours in a scalar field (i.e, LAVD). This computational task belongs to the field of image processing and is amenable to multi-core parallelization, and less demanding in comparison to other Lagrangian vortex identification methods age from the LAVD scalar field. A pixel gives the value of the corresponding particle's LAVD. The global domain we employ for this study spans all $360^{\circ}$ in longitude and from $80^{\circ} \mathrm{S}$ to $64^{\circ} \mathrm{N}$ in latitude. This domain is covered with a Lagrangian particle mesh at a uniform resolution of $1 / 32^{\circ}$, producing an LAVD ical geometry when computing areas and distances by using the appropriate local tangent plane projections.)

Identifying local maxima and contours in an image requires the introduction of two free parameters: a parameter to control how many local maxima for identifying contours. Similar parameters are also found in many Eulerian sea surface anomaly methods (Chelton et al., 2007; Frenger et al. 2015); the fundamental difference is that our underlying field is derived from Lagrangian 
quantities. For this study, we inform our determination of these parameters by is required to construct the basic circular velocity field pattern of a vortex as depicted in Figure 4. Accordingly, we intend to identify coherent vortices with 
length scales greater than or equal to the length of two grid cells (which varies 4 array of particles in the interior of each nominal $0.1^{\circ} \times 0.1^{\circ}$ velocity cell as shown in Figure 4. In this work, we advect the Lagrangian particles in the daily mean surface currents from the pre-industrial CM2.6 simulation of 200 years. Advection using archived velocity fields is conducted with the MITgcm 235 Abernathey \& Marshall (2013). Trajectories are integrated using a 4-th order Runge-Kutta scheme with a timestep of 900.0 seconds.

Velocity fields are interpolated from the Arakawa B-grid of MOM5 to an 


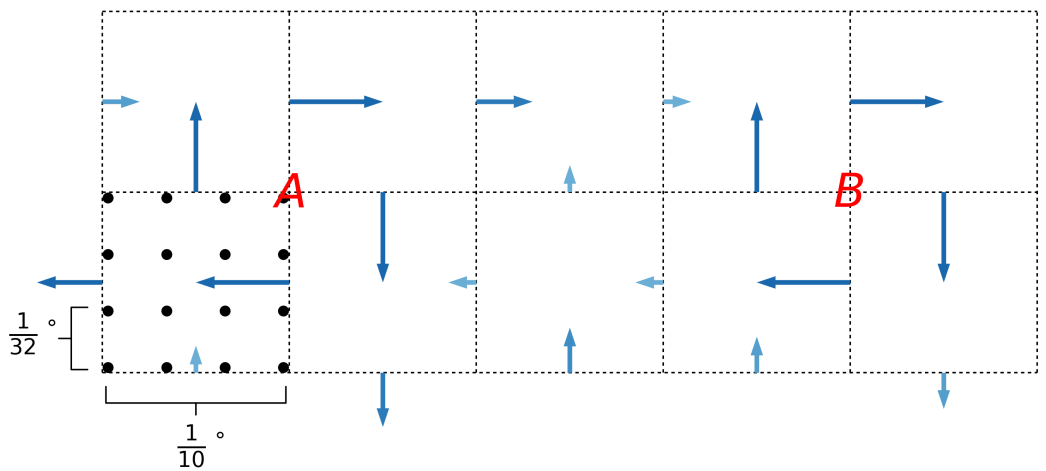

Figure 4: Schematic depicting a horizontal velocity field containing two neighboring grid-scale vortices (with centers A and B) on a Southwest convention Arakawa C-grid. Each vortex exhibits a four-cell clock-wise circular flow pattern. A sample of the Lagrangian particle mesh (shown as black points) is displayed in the lower leftmost grid cell. The vortex centers are three grid cells from each other, which corresponds to a separation of roughly ten Lagrangian particles.

Arakawa C-grid required for the MITgcm (Arakawa \& R. Lamb, 1977). The MOM5 B-grid is based on the Murray Tripolar grid of Murray (1996), which has a standard quasi-mercator latitude by longitude format south of $65^{\circ} \mathrm{N}$, but contains a non-spherical grid north of $65^{\circ} \mathrm{N}$ with singularities over Northern Canada and Northern Russia (Griffies, 2012). To avoid the complexities associated with interpolating from the non-spherical polar region of the B-grid to a spherical C-grid, the domain is truncated at $65^{\circ} \mathrm{N}$. The Lagrangian particle mesh covers this near-global domain at $1 / 32^{\circ}$ resolution with 37 million particles. The LAVD and position of each Lagrangian particle is output and saved daily. For example, Figure 3 exhibits particle trajectories after 60 days of advection.

For a 30-day interval, we distribute the advection of the global particle mesh in MITgcm over 128 processors. This computation takes an hour and produces 
$32 \mathrm{~Gb}$ of trajectory data.

\section{Results}

We proceed to analyze RCLV identified in CM2.6 and walk through how occupies 4 grid cells, we expect a minimum separation of at least three grid cells between LAVD maxima at the respective centers of two neighboring RCLVs as illustrated in Figure 4. Interpreting this criterion in terms of the nominal $4 \times$ 

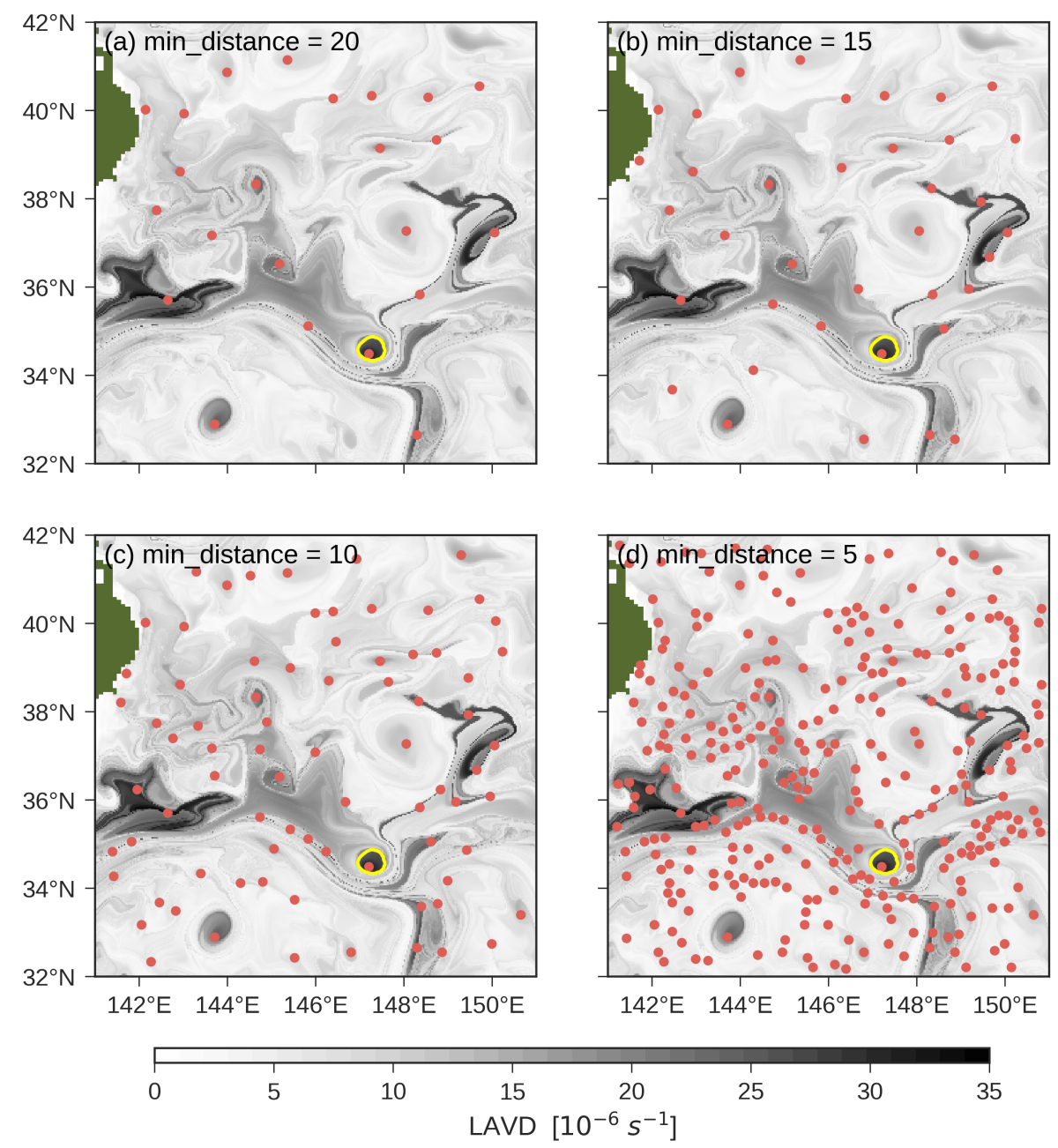

Figure 5: Kuroshio LAVD field with the local maxima (overlain in red scatter points) from identification with min_distance choices of a) 20, b) 15, c) 10, d) 5 pixels. The RCLV search in all cases identifies a single vortex whose boundary (shown as yellow contour). 

exists for every value in the interval between the minimum and maximum LAVD pixel values in the image. To make progress, we are required to specify a fixed LAVD increment with which to iterate through the nested contour field. This contour increment introduces another free parameter to the method. Figure 305 contour increment of $4 \times 10^{-7} \mathrm{~s}^{-1}$ was employed to produce this contour field.

A coarse LAVD contour increment will distort the geometry of the LAVD field, giving rise to a problematic coupling between the convexity deficiency and the contour increment. We desire that the effects of varying the free parameters 


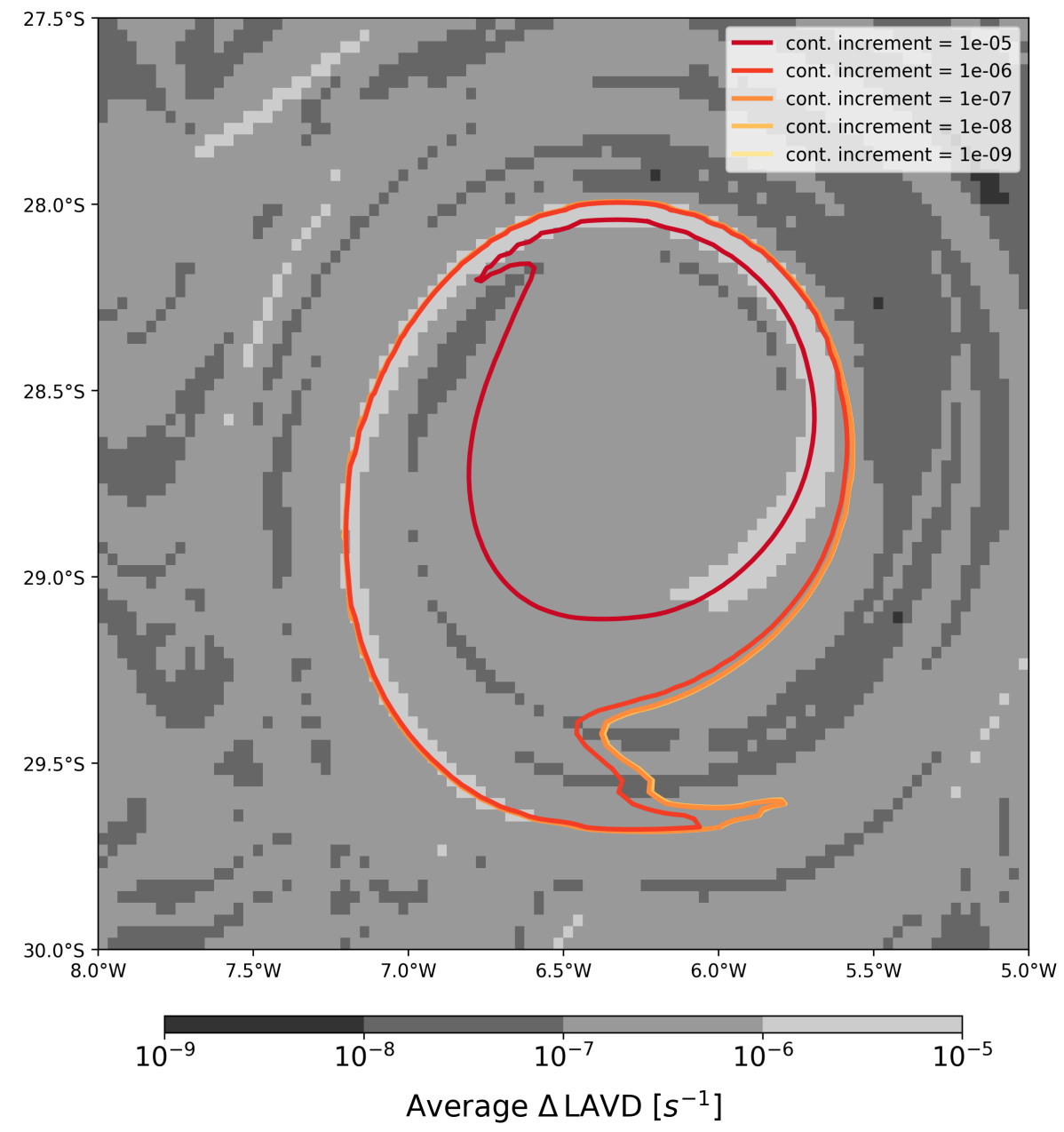

Figure 6: Average difference in LAVD between a pixel and its contiguous neighbors, computed for the LAVD field shown in Figure 2 Contours (shown sequentially from red to yellow) are the RCLV identification results for various choices of contour increment. Convergence occurs with a contour increment of $10^{-8} \mathrm{~s}^{-1}$. 
Arriving at a contour increment that balances the competing interests (resolving the contour convexity vs. minimizing computation time) requires understanding how the LAVD varies from pixel to pixel. We assess this variation by, for instance, taking the average of the difference between the LAVD value at a pixel and the LAVD value at its four neighboring pixels:

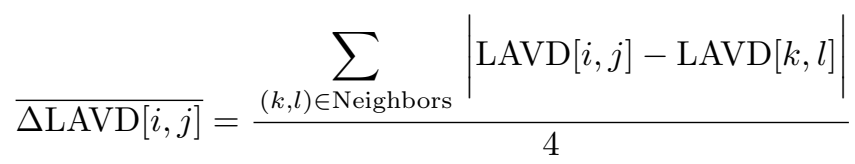

where $\mid$ is the absolute value and the sum runs over the indices of the contiguous pixels, Neighbors $=\{(i+1, j),(i-1, j),(i, j+1),(i, j-1)\}$. Figure 6 presents this averaged difference field for the Agulhas LAVD field previously plotted in Figure 2 With the exception of fewer than ten pixels in the local 128 by 128 pixel domain, the pixel-to-pixel variation in LAVD exceeds $10^{-8} \mathrm{~s}^{-1}$. We thus expect that a contour increment of $10^{-8} \mathrm{~s}^{-1}$ appropriately resolve the grid-scale RCLV features.

We verify this hypothesis by conducting a sensitivity analysis in which we compare RCLV boundaries identified with varying choices of contour increment. is achieved with a contour increment between $10^{-7}$ to $10^{-8} \mathrm{~s}^{-1}$. On a global scale, we tested the robustness of the contour increment choice by comparing identification with a contour increment of $10^{-8} \mathrm{~s}^{-1}$ to $10^{-9} \mathrm{~s}^{-1}$. Both resultant datasets contained the same number of vortices and there was less than a $1 \%$ difference in cumulative area between the two datasets. As a result, we have employed a contour increment of $10^{-8} \mathrm{~s}^{-1}$ in this paper. For reference, RCLV detection with floater on a global LAVD image with a contour increment of 
$10^{-8} \mathrm{~s}^{-1}$ takes roughly 3 hours.

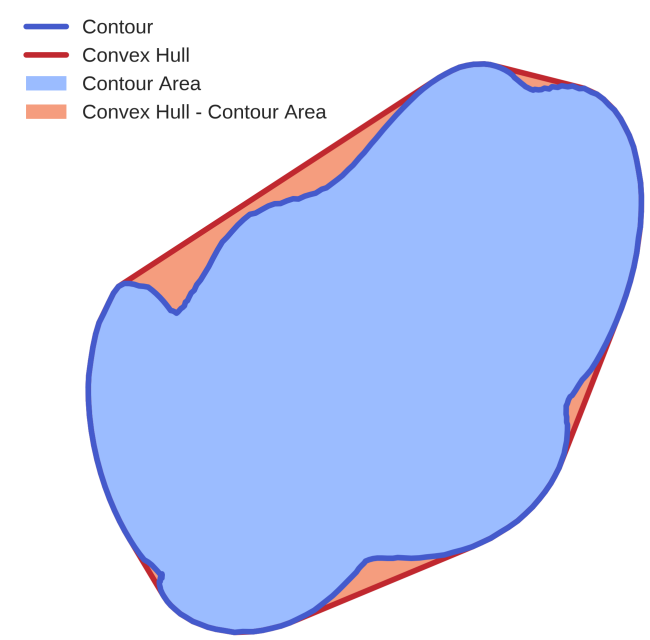

Figure 7: Schematic of a contour surrounded by its convex hull. The convexity deficiency equals the difference in area between the convex hull and the contour (orange region) divided by the contour's area (blue region). For the above contour, the convexity deficiency equals 0.06 .

\subsection{Convexity Deficiency}

For the LAVD method, the fundamental tuning question is how to determine the boundary of the RCLV using knowledge of the LAVD contours' convexity. To this end, we utilize the convexity deficiency, abbreviated as CD, as a measure of the convexity of a 2D LAVD contour. CD is equal to the area between the contour and its convex hull, divided by the area enclosed by the contour (Haller et al. 2016). The convex hull of a curve is the smallest convex set that contains the curve as shown in Figure 7. If a curve has a small value of CD, then the curve is nearly convex.

While plots in the vein of Figure 3 evince a sound connection between LAVD contours and material coherency, they do not directly address the question of how to determine the convexity deficiency parameter. This question is particularly important because the RCLV identification results are highly sensitive 


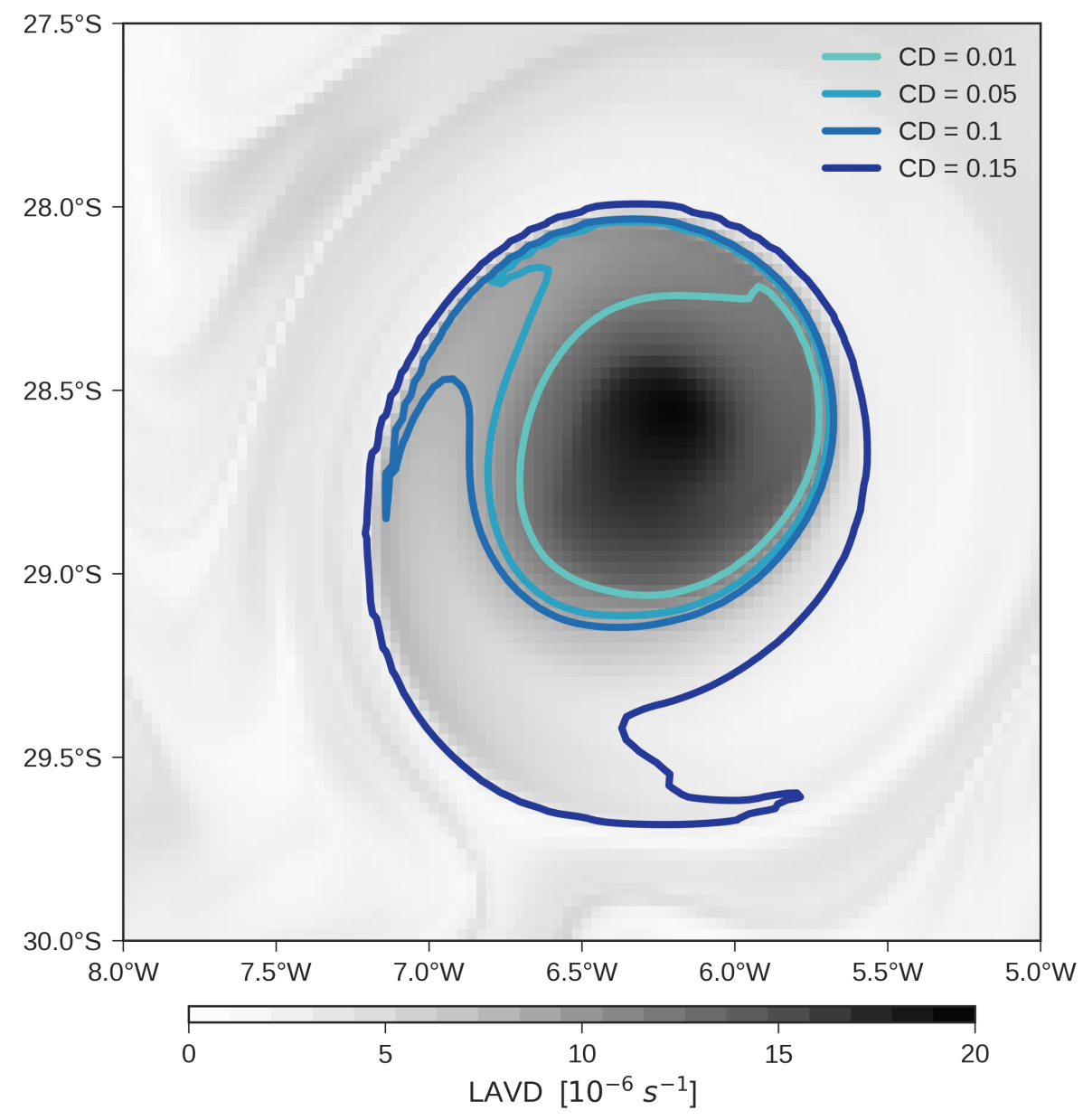

Figure 8: LAVD field computed over a 30-day period in the Agulhas region. Contours (shown in blue) are the RCLV identification results for various choices of CD, all encircling an Agulhas ring (formerly shown in Figure 22. 
to variations in CD. This sensitivity is demonstrated in Figure 8 for the RCLV detection of an Agulhas ring.

Varying CD from .01 to .15 results in a concentric set of RCLV boundaries herent a feature must be in order to merit distinguishing it from the turbulent environmental fluid as a "coherent structure". To avoid this subjectivity, the idea of simply setting $\mathrm{CD}=0$ and eliminating it as a free parameter may sound appealing. The consequence is that RCLV boundaries are strictly convex polytory: we have merely transformed the ambiguity in determining CD into an 

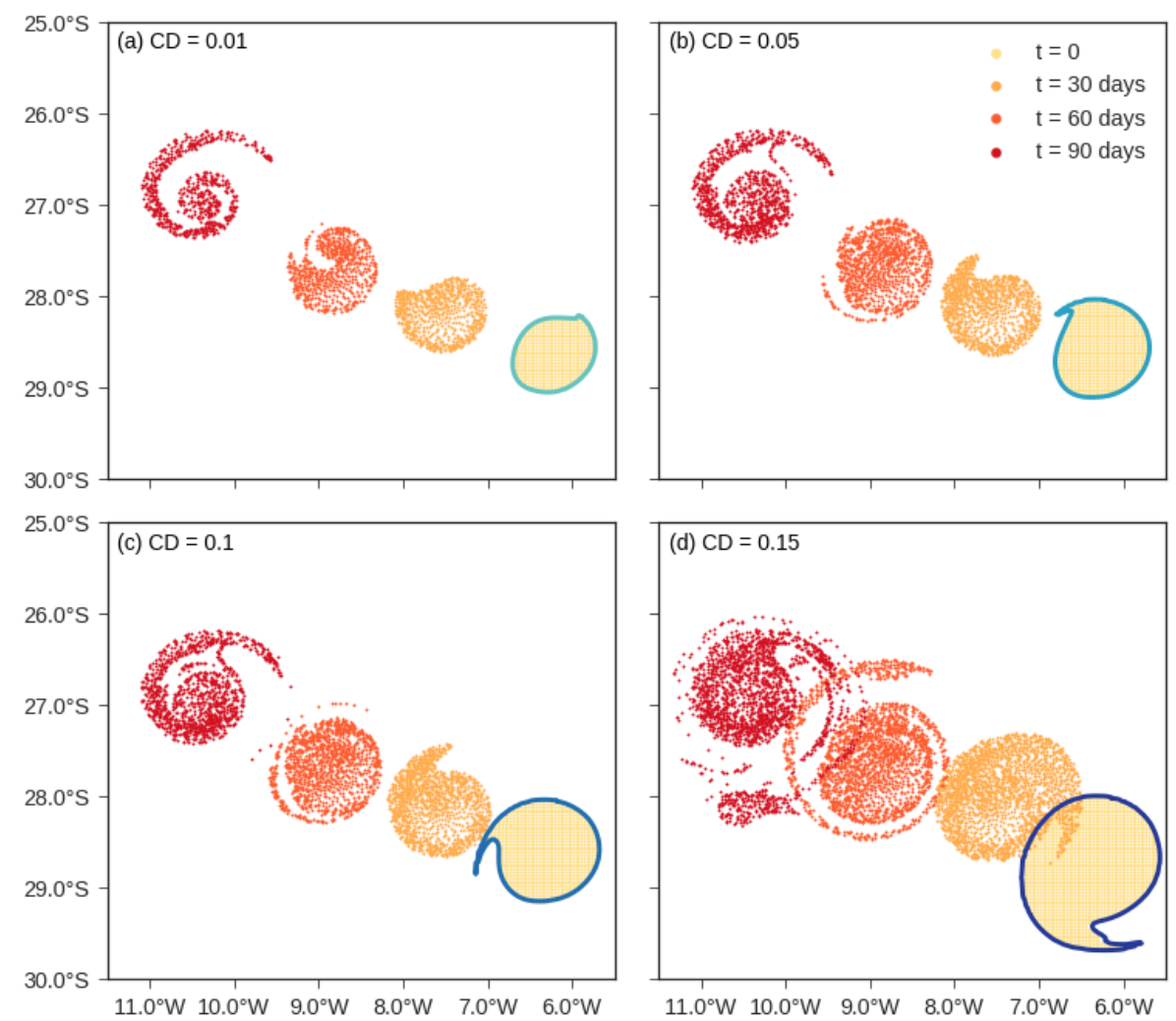

Figure 9: Evolution of RCLVs shown in Figure 8 for parameter choices of a) CD $=.01, \mathrm{~b}$ ) $\mathrm{CD}=.05, \mathrm{c}) \mathrm{CD}=0.1, \mathrm{~d}) \mathrm{CD}=0.15$. RCLVs were identified using only the first 30 days of trajectory data. Later trajectories are shown to demonstrate the vortex decay process. See text for discussion.

ambiguity in choosing the Lagrangian mesh resolution.

Perhaps part of the uncertainty here is due to the somewhat ill-defined nature of a coherent structure's boundary. This ambiguity is even present in McWilliams' original work on 2D quasi-geostrophic turbulence, where he steers clear of quantifying the boundary and a precise distinction between vortex and environment is challenging (McWilliams, 1984). Recently, Wang et al. (2016) studied the life cycle of an Agulhas ring using Lagrangian-based vortex detection and showed that periodic "coherence regain" events, during which the vortex 

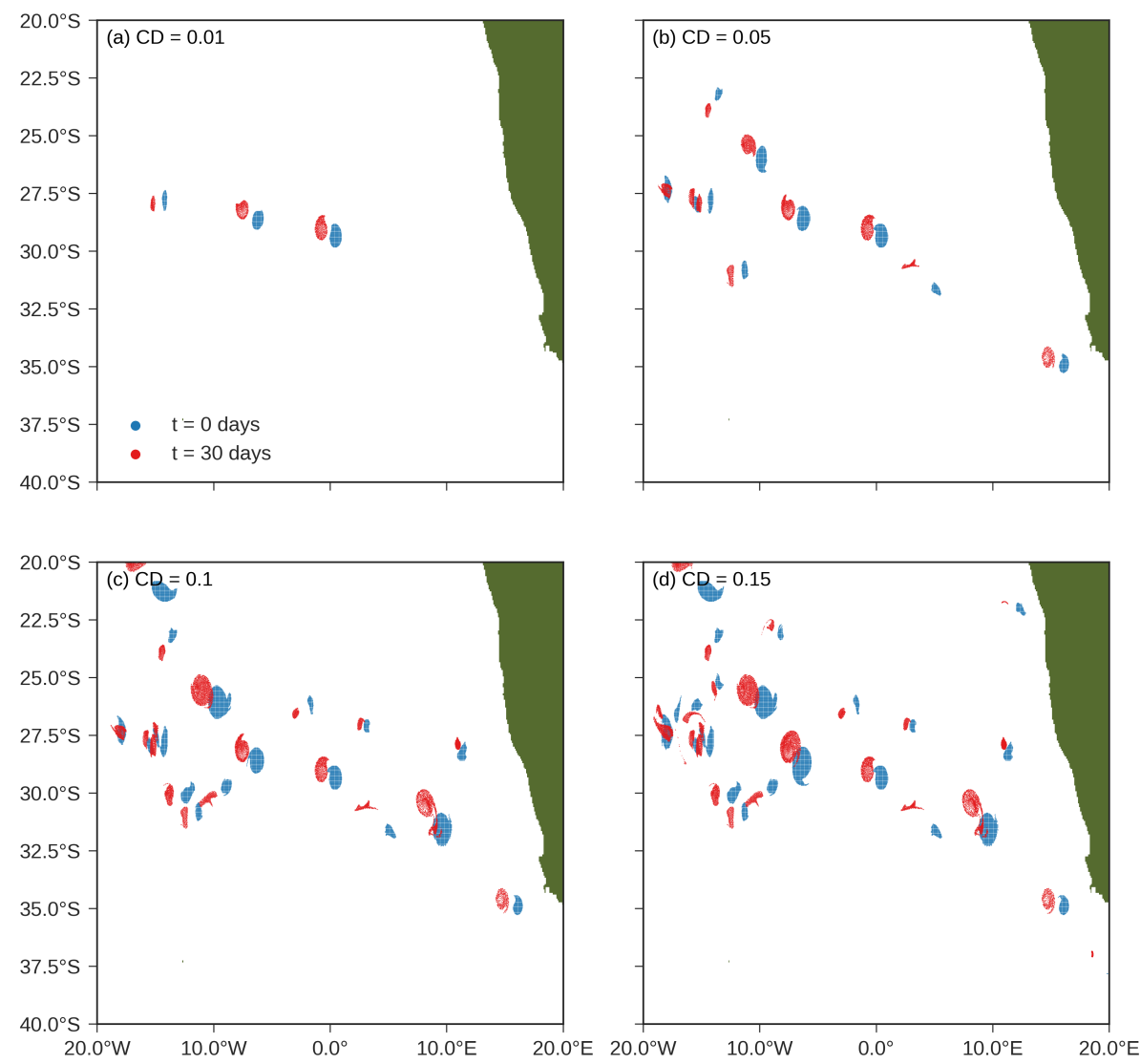

Figure 10: Initial locations (shown in blue) and final locations (shown in red) of 30-day RCLVs in the Agulhas region of the South Atlantic Ocean. RCLVs are identified with parameter choices of a) $\mathrm{CD}=.01, \mathrm{~b}) \mathrm{CD}=.05, \mathrm{c}) \mathrm{CD}=0.1, \mathrm{~d}) \mathrm{CD}=0.15$.

entrains surrounding fluid, complicate this distinction between environment and vortex.

This ambiguity in the definition of a coherent structure's boundary-along with the acute sensitivity of the method to CD-motivates the idea of not settling on a single value of $\mathrm{CD}$. Rather, we argue that it is appropriate and desirable to leverage the sensitivity to CD in order to separate RCLVs into three broad classes: strictly coherent, moderately coherent, and weakly coherent (aka 
leaky). In order to accomplish this goal, we need to choose three representative $\mathrm{CD}$ values for each coherency class. While there is some subjectivity in choosing representative $\mathrm{CD}$ values, this setup is arguably better motivated than the alternative of simply choosing a single CD for all of the analysis. This approach also has the advantage of revealing dynamical insight on the geographical distribution of coherency in the ocean. For instance, it may be valuable to know what regions form leaky vortices exclusively or which regions experience a high frequency of strict coherent vortex formation.

\subsection{Coherency Index}

To achieve a coherency categorization based on $\mathrm{CD}$, we have to make precise the relationship between material coherency and CD. To this end, we introduce a Coherency Index. To quantify an RCLV's spatial compactness, we compute the variance of the particle positions,

$$
\sigma^{2}(t)=\left\langle|\boldsymbol{X}(t)-\langle\boldsymbol{X}(t)\rangle|^{2}\right\rangle
$$

where \langle\rangle indicates an average over the set of RCLV particles and || is the standard Euclidean distance. A coherent vortex remains spatially compact under advection. To assess the change in spatial compactness over a time interval $[0, t]$, we compute the Coherency Index (CI),

$$
\mathrm{CI}=\frac{\sigma^{2}(0)-\sigma^{2}(t)}{\sigma^{2}(0)} .
$$

The sign and magnitude of CI specify the material coherency of an RCLV. By construction, CI is positive for vortices that are strongly coherent, with a theoretical limit of $\mathrm{CI}=1$ for vigorously downwelling vortices that contract to a point (as in Figure 3). For a weakly coherent vortex that sheds material, $\sigma^{2}(t)$ can be arbitrarily larger than $\sigma^{2}(0)$, and CI becomes arbitrarily negative. Figure 11 presents sample vortex motions and their associated CIs. The vortices were selected from the vortex field discussed earlier in panel d) of Figure 10. As we might expect, the CI metric penalizes a vortex for dispersing and developing filaments, and rewards a vortex for growing more compact. We also observe 

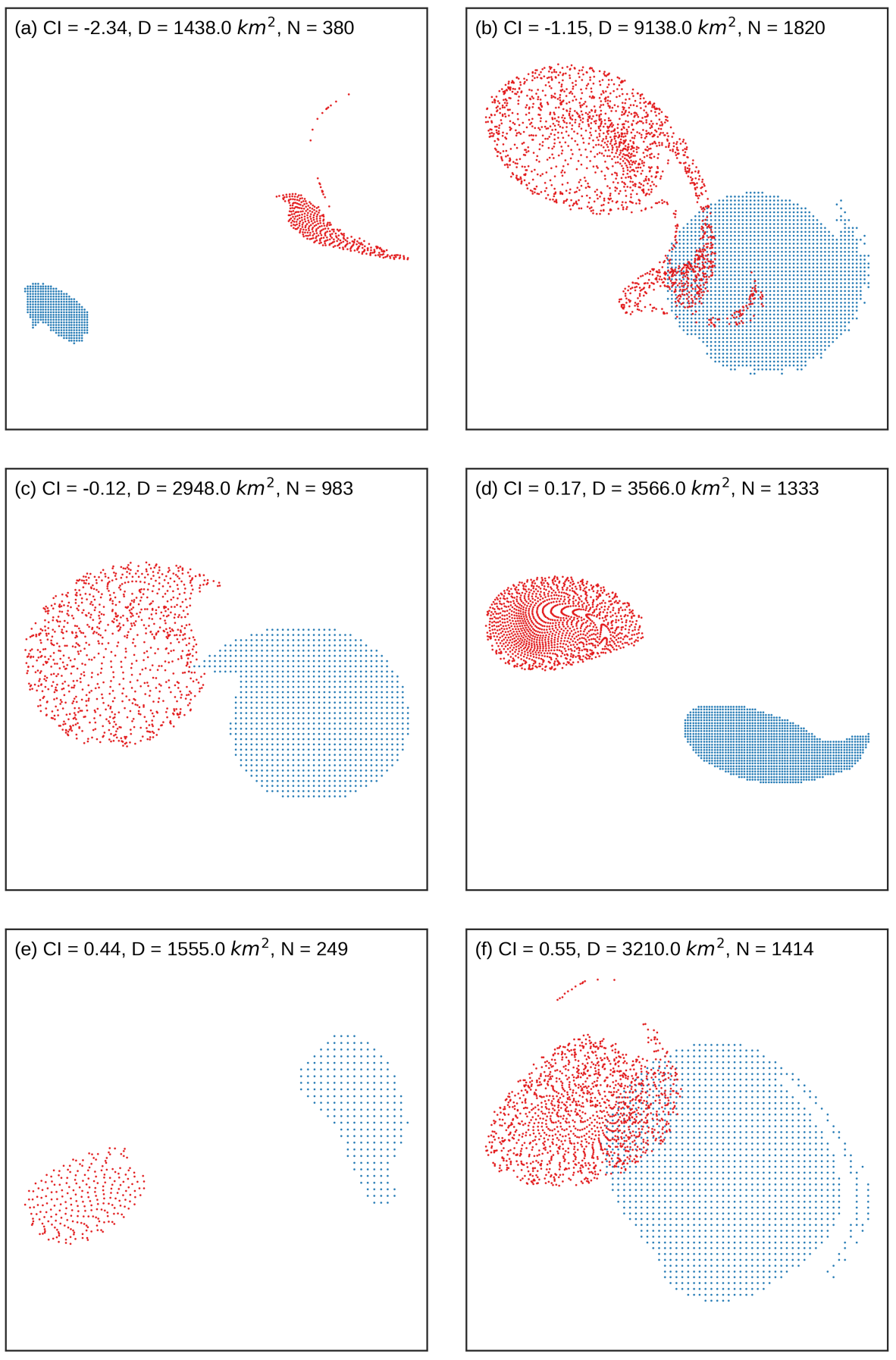

Figure 11: Values of Coherency Index (CI) for representative RCLVs, which were selected from the vortex field shown in panel d) of Figrzire 10 Rows demonstrate examples of leaky, coherent, and contracting vortices respectively. Initial locations of trajectories are shown in blue and final locations are shown in red. The dispersion (D) and number of particles (N) (directly proportional to the vortex's area) are included to illustrate the explanation of why the dispersion is a poor coherency metric. 
that CI values close to zero correspond to vortices whose movements closely resemble solid body motion.

In prior literature, the variance of Lagrangian particle displacements has been used to quantify how sets of particles spread out over time (see the review of LaCasce (2008), and references therein). This quantity, known as the dispersion, is given by

$$
D(t)=\left\langle|\boldsymbol{X}(t)-\boldsymbol{X}(0)-\langle\boldsymbol{X}(t)-\boldsymbol{X}(0)\rangle|^{2}\right\rangle .
$$

Note that $D(t)$ measures how the particles disperse over the time interval $[0, t]$, while $\sigma^{2}(t)$ measures only the instantaneous spatial distribution of the particles at a fixed time t. We now explain several reasons why $D(t)$ has limited utility as a coherency metric.

First, we note the problematic dependence of $D(t)$ on the vortex's area, which governs the spatial scale of the displacements. The same value of $D(t)$ can correspond to a fairly coherent large vortex and to a leaky small vortex. This undesirable feature of $D(t)$ is demonstrated in Figure 11. A similar point is illustrated in Figure 2 of LaCasce (2008) with the conclusion being that "[w]hile the dispersion reflects the clouds size, it is fairly insensitive to the clouds distribution in space".

Another fundamental limitation of $D(t)$ is the quantity's inability to distinguish between radial expansion versus contraction. Consider a particle cloud with circular symmetry that is radially expanded or contracted. Since the vortex maintains its circular shape, the mean displacement $(\langle\boldsymbol{X}(t)-\boldsymbol{X}(0)\rangle)$ is zero by symmetry. The value of $D(t)$ is then set by only the magnitude of the change in the vortex's radius. $D(t)$ is ignorant of whether the change in the vortex's radius is positive or negative, and thus cannot distinguish between expansion and contraction. Therefore, $D(t)$ overlooks a signature difference between upwelling and downwelling vortices. These limitations of $D(t)$ are not shared by CI, which is independent of vortex area and capable of distinguishing expansion from contraction.

To further understand the relationship between CI and the convexity defi- 


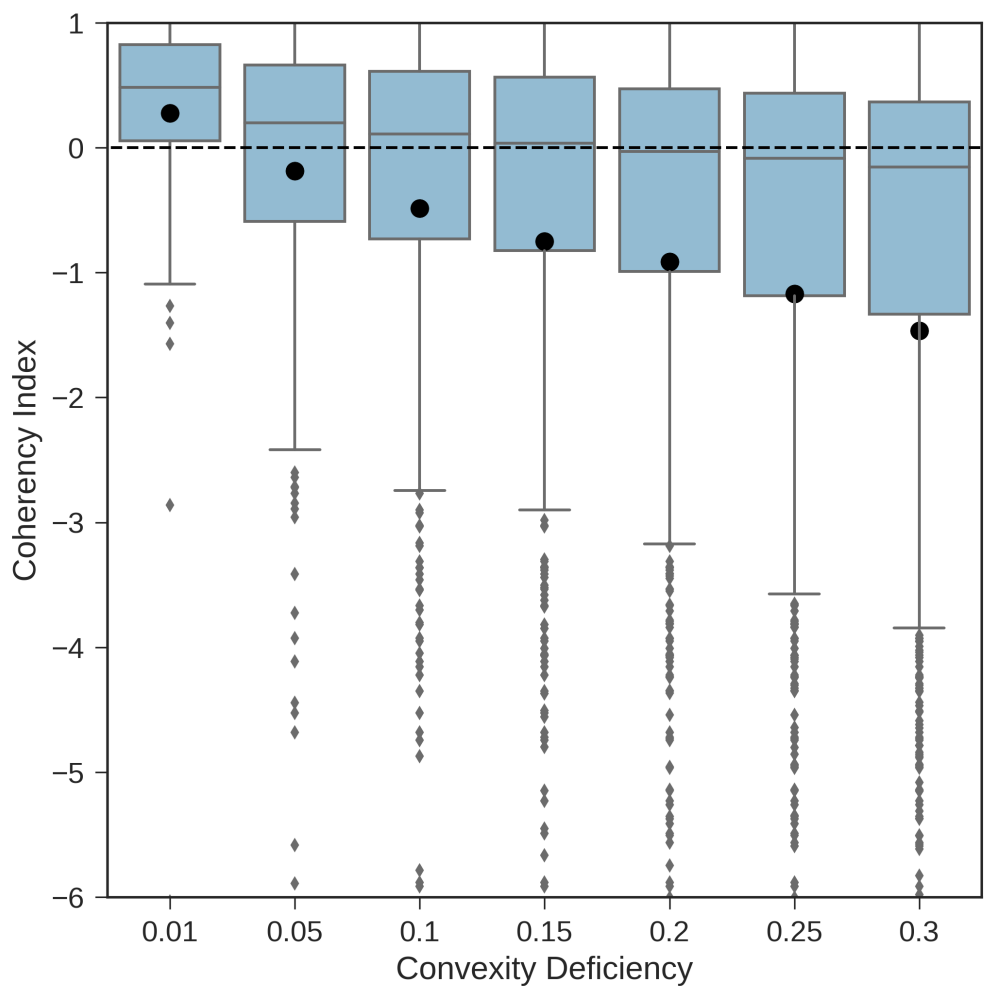

Figure 12: A box-and-whiskers plot of the Coherency Index distributions for RCLV datasets resulting from identification with various choices of convexity deficiency parameter. For each distribution, the mean is indicated by a black point, the second (third) quartile is contained in the lower (upper) box, and the standard deviation is displayed as a whisker. Data farther than one standard deviation from the mean is plotted discretely. RCLVs were identified across the full global domain using 30-day trajectories. To put the values of Coherency Index in context, see Figure 11. Also see text for further discussion.

ciency, we conduct a global identification of RCLVs for various choices of convexity deficiency. We then compute and compare the Coherency Index distributions. All calculations are done with a global 30-day LAVD field. The results are summarized in the table below and displayed in Figure 12 . Note that a limited spatial region of this global data set was previously shown in Figure 10 . 


\begin{tabular}{|l|l|l|l|}
\hline & Number & Mean CI & Median CI \\
\hline $\mathrm{CD}=.01$ & 109 & 0.28 & 0.48 \\
$\mathrm{CD}=.05$ & 346 & -0.18 & 0.20 \\
$\mathrm{CD}=0.1$ & 579 & -0.48 & 0.11 \\
$\mathrm{CD}=0.15$ & 789 & -0.74 & 0.03 \\
$\mathrm{CD}=0.2$ & 1014 & -0.91 & -0.03 \\
$\mathrm{CD}=0.25$ & 1250 & -1.17 & -0.08 \\
$\mathrm{CD}=0.3$ & 1534 & -1.47 & -0.16 \\
\hline
\end{tabular}

The clear trend aligns with our expectations: the higher the value of convexity deficiency, the greater the number of identified leaky vortices. This fact is reflected by the Coherency Index distributions medians, means, and quartiles 445 all shifting uniformly down the $\mathrm{y}$-axis towards negatives CI values in Figure 12

First, we observe that for the strictest convexity deficiency threshold of $\mathrm{CD}=$ .01 , the mean convexity index is positive, indicating that the method is primarily identifying coherent objects that grow more compact with time. At $\mathrm{CD}=.05$, the mean value of the convexity index changes sign to -.18 . This signals a coherency regime change: although the median is still positive, there are now many dispersive RCLVs in the dataset. All subsequent larger values of CD intensify the leakiness and shift the mean further negative. The key point here is that to classify the strictly coherent RCLVs we should choose a CD $<.05$.

The next shift in coherency regime occurs between $\mathrm{CD}=0.15$ and $\mathrm{CD}=0.2$. In this interval, the median changes sign, revealing that at $\mathrm{CD}=0.2 \mathrm{most}$ identified vortices are leaky. The proximity of the mean to the 1st quartile of data also reflects the presence of very incoherent vortices. Since most vortices leak and some do so to an extreme degree, we conclude that between $\mathrm{CD}=0.15$ and $\mathrm{CD}=0.2$ we transition to the weakly coherent regime. These findings suggest that we employ parameter choices of $\mathrm{CD}=.01, \mathrm{CD}=0.1$, and $\mathrm{CD}=$ 0.25 to survey the three different types of vortices.

In summary, we delineated between the leaky and moderately coherent regimes by when the mean Coherency Index is approximately zero. We then 
defined the end of the moderately coherent by when the median Coherency Index is approximately zero. Sample results for the $C D$ values corresponding to these definitions are shown in Figure 13 for a domain in the Pacific Ocean. The varying dispersive nature of the identified vortices is in agreement with our expectations based on the above definitions.

The broad fluctuation in the results emphasizes the importance of understanding the method's sensitivity and how it relates to the physics of the identified vortices before applying the method to address vortex census questions. Importantly, we find an order of magnitude more leaky vortices than strictly coherent vortices. Censuses conducted with $\mathrm{CD}=.01$ versus $\mathrm{CD}=0.25$ would consequently reach RCLV net transport estimates that differ by at least an order 475 of magnitude.

For comparison, we have included panel a) of Figure 14 to demonstrate the dispersive nature of randomly selected patches of fluid. The control results are characterized by a CI distribution with an approximate mean of -7 and median of -5 .

\section{Discussion and Conclusions}

The above analysis demonstrated that the LAVD method results are highly sensitive to variations in the free parameters: convexity deficiency, min_distance, and contour increment. We also described, however, that this sensitivity can be physically rationalized, enabling the oceanographer to utilize the method to reveal objective structures. Through convergence analysis, we have demonstrated that the full mesoscale vortex field can be detected with min_distance $=10$, and that the nested LAVD contour fields can be fully resolved with a contour increment of $10^{-8} \mathrm{~s}^{-1}$. Subsequently, we introduced a Coherency Index to quantify the coherent versus dispersive nature of the identification results. This diagnostic enabled us to select tunings of the convexity deficiency that explore the entire spectrum of coherency in the ocean. In particular, we showed that convexity deficiency choices of $.01,0.1$, and 0.25 are representative values for 

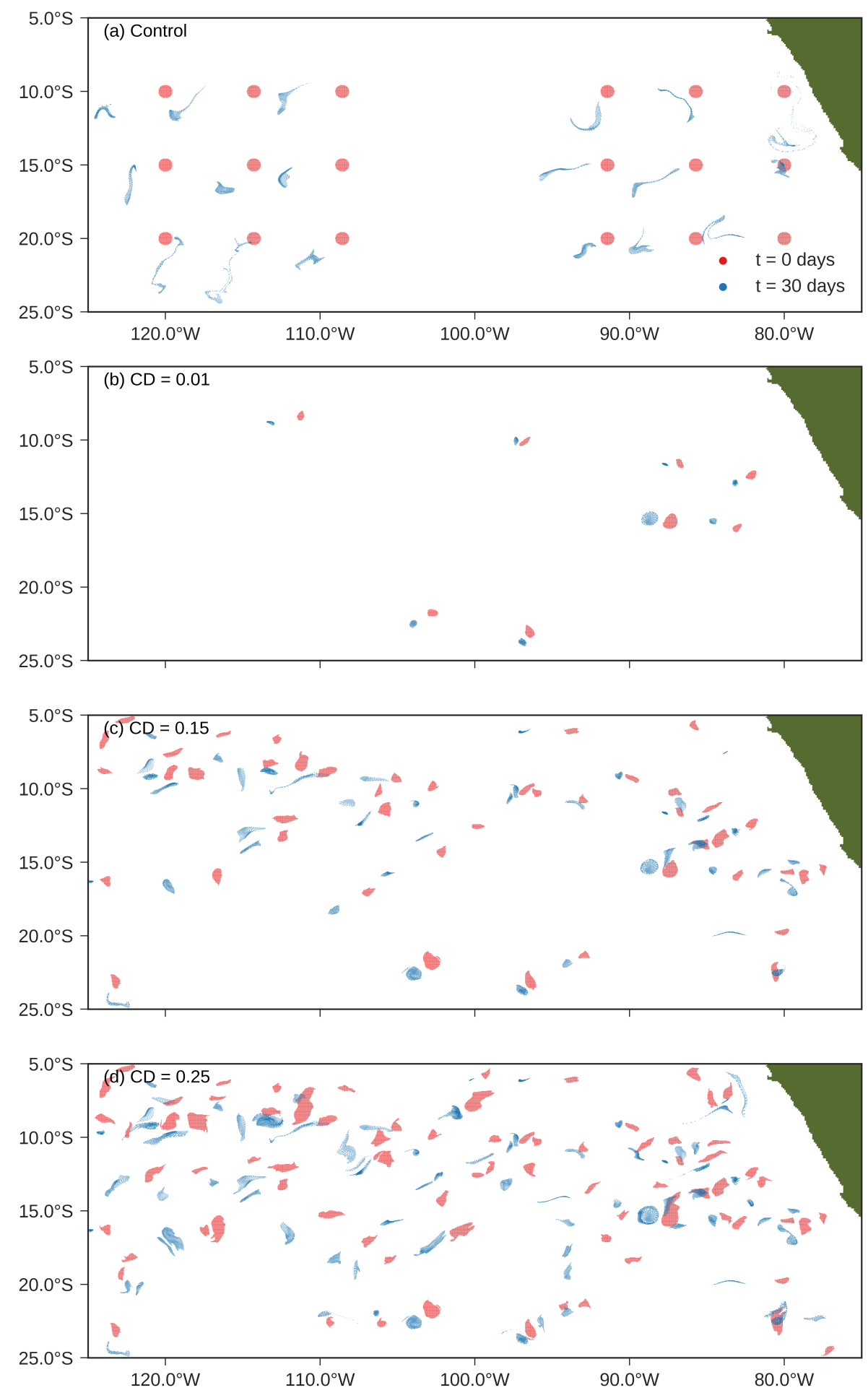

30

Figure 13: a) trajectories of two randomly selected arrays of control regions, and RCLV results for $\mathrm{b}) \mathrm{CD}=.01$; $) \mathrm{CD}=0.1 ; \mathrm{d}) \mathrm{CD}=0.25$ on a common subdomain in the Pacific Ocean. See text for discussion. 
strictly, moderately, and weakly coherent vortices. Note that because resolution and dynamics vary from model to model, these exact values may only be appropriate for our specific model dataset. However, the tuning methodology developed here should be equally effective and relevant for applying the method to other models and observations. Furthermore, the coherency index provides a means to compare and assess results across models and even identification methods. Forthcoming work builds on the foundation laid in this paper, and presents the results from a global census of vortices for each coherency class in CM2.6.

Our analysis was limited to the two-dimensional surface manifestation of the vortex flow, but coherent vortices are, of course, fully three dimensional structures. The significant computational challenge posed by the $3 \mathrm{D}$ problem tices that show no signs of convergence/divergence (i.e. CI $\approx 0$ ) as in Figure 11 , d. For downwelling vortices, however, the horizontal motion of a surfaceconstrained Lagrangian particle may differ significantly from the horizontal motion of the fully 3D fluid trajectory. Consider the downwelling vortex of Figure ${ }_{520} 3$. The vortex has an initial horizontal surface area of $3.5 \times 10^{4} \mathrm{~km}^{2}$, and the depth of the ocean model's surface grid cell is $10 \mathrm{~m}$, giving a total initial volume of $350 \mathrm{~km}^{3}$ in the surface layer. After 60 days of advection, this material has been compressed to a narrow core with an area of $430 \mathrm{~km}^{2}$. Consequently, we 
infer that the vortex downwelled over the 60 day interval with a vertical volume using a root finding algorithm. Root finding would eliminate the need to specify a fixed contour increment. Efforts are underway to implement this in floater (available at https://github.com/rabernat/floater) and explore it as an alternative means of searching the contour field.

\section{5. Acknowledgment}

NT would like to thank Spencer Clark for his Python expertise and helpful discussions. The authors thank Laure Zanna and Sonya Legg for discussion and comments. We thank four anonymous reviewers whose input has improved the manuscript. NT acknowledges support from U.S. Department of Energy under Award DE-SC0012457. CD and NT acknowledge support from National Aeronautics and Space Administration (NASA) under Award NNX14AL40G. RPA acknowledges support from an NSF CAREER award (OCE 1553593) and 
NASA award NNX16AJ35G.

\section{References}

口 in the eastern pacific. J. Phys. Oceanogr., 48, 667-685. doi 10.1175/ JPO-D-17-0102.1.

Abernathey, R., \& Marshall, J. C. (2013). Global surface eddy diffusivities derived from satellite altimetry. J. Geophys. Res., 118, 901-916.

Adcroft, A., Hill, C., Marshall, J. et al. (2014). MITgcm user manual.. MIT

Department of EAPS Cambridge. URL: http://mitgcm.org/public/r2_ manual/latest/online_documents/manual.html.

Arakawa, A., \& R. Lamb, V. (1977). Computational design of the basic dynamical processes of the ucla general circulation model, . 17 .

Beron-Vera, F. J., Olascoaga, M. J., Haller, G., Farazmand, M., Triñanes, J., \& Wang, Y. (2015). Dissipative inertial transport patterns near coherent lagrangian eddies in the ocean. Chaos, 25, 087412.

Burden, R. L., \& Faires, J. D. (1985). Numerical analysis. PWS Publishers.

Chelton, D. B., Schlax, M. G., \& Samelson, R. M. (2011). Global observations of nonlinear mesoscale eddies. Prog. Oceanogr., 91, 167-216.

Chelton, D. B., Schlax, M. G., Samelson, R. M., \& de Szoeke, R. A. (2007). Global observations of large oceanic eddies. Geophys. Res. Lett., 34.

Delworth, T. L., Rosati, A., Anderson, W., Adcroft, A. J., Balaji, V., Benson, R., Dixon, K., Griffies, S. M., Lee, H.-C., Pacanowski, R. C. et al. (2012). Sim575 ulated climate and climate change in the gfdl $\mathrm{cm} 2.5$ high-resolution coupled climate model. J. Clim., 25, 2755-2781.

Dong, C., McWilliams, J. C., Liu, Y., \& Chen, D. (2014). Global heat and salt transports by eddy movement. Nat. Commun., 5, 3294. 
Dufour, C. O., Griffies, S. M., de Souza, G. F., Frenger, I., Morrison, A. K.,

Palter, J. B., Sarmiento, J. L., Galbraith, E. D., Dunne, J. P., Anderson, W. G., \& Slater, R. D. (2015). Role of mesoscale eddies in cross-frontal transport of heat and biogeochemical tracers in the southern ocean. J. Phys. Oceanogr., 45, 3057-3081. doi 10.1175/JP0-D-14-0240.1

Dufour, C. O., Morrison, A. K., Griffies, S. M., Frenger, I., Zanowski, H., \& Winton, M. (2017). Preconditioning of the weddell sea polynya by the ocean mesoscale and dense water overflows. J. Clim., 30, 7719-7737.

dOvidio, F., De Monte, S., Della Penna, A., Cotté, C., \& Guinet, C. (2013). Ecological implications of eddy retention in the open ocean: a lagrangian approach. J. Phys. A, 46, 254023.

Frenger, I., Münnich, M., Gruber, N., \& Knutti, R. (2015). Southern ocean eddy phenomenology. J. Geophys. Res.: Oceans, 120, 7413-7449.

Froyland, G., Padberg, K., England, M. H., \& Treguier, A. M. (2007). Detection of coherent oceanic structures via transfer operators. Phys. Rev. Lett., 98, 224503 .

${ }_{595}$ Goddard, P. B., Dufour, C. O., Yin, J., Griffies, S. M., \& Winton, M. (2017). Co2-induced ocean warming of the antarctic continental shelf in an eddying global climate model. J. Geophys. Res.: Oceans, .

Griffies, S. M. (2012). Elements of the Modular Ocean Model (MOM): 2013 release (GFDL Ocean Group Technical Report No. 7). Princeton, USA: NOAA/Geophysical Fluid Dynamics Laboratory. $614+$ xiii pages.

Griffies, S. M., Winton, M., Anderson, W. G., Benson, R., Delworth, T. L., Dufour, C. O., Dunne, J. P., Goddard, P., Morrison, A. K., Rosati, A. et al. (2015). Impacts on ocean heat from transient mesoscale eddies in a hierarchy of climate models. J. Clim., 28, 952-977.

605

Hadjighasem, A. (2016). Lagrangian-averaged-vorticity-deviation-

a lavd. Github Repository. URL: https://github.com/LCSETH/ 
Hadjighasem, A., Farazmand, M., Blazevski, D., Froyland, G., \& Haller, G. (2017). A critical comparison of lagrangian methods for coherent structure detection. Chaos, 27, 053104.

Hadjighasem, A., Karrasch, D., Teramoto, H., \& Haller, G. (2016). Spectral-

¿ clustering approach to lagrangian vortex detection. Phys. Rev. E, 93. doi:10. 1103/physreve.93.063107.

Haller, G. (2016). Dynamic rotation and stretch tensors from a dynamic polar decomposition. J. Mech. and Phys. Solids, 86, 70-93.

Haller, G., \& Beron-Vera, F. J. (2013). Coherent lagrangian vortices: the black holes of turbulence. J. Fluid Mech., 731, R4. doi:10.1017/jfm.2013.391.

Haller, G., Hadjighasem, A., Farazmand, M., \& Huhn, F. (2016). Defining coherent vortices objectively from the vorticity. J. Fluid Mech., 795, 136173.

Haller, G., \& Sapsis, T. (2008). Where do inertial particles go in fluid flows? Physica D, 237, 573 - 583. doi:10.1016/j.physd.2007.09.027.

Hughes, C. W., \& Miller, P. I. (2017). Rapid water transport by long-lasting modon eddy pairs in the southern midlatitude oceans. Geophys. Res. Lett., 44. doi:10.1002/2017gl075198.

LaCasce, J. (2008). Statistics from lagrangian observations. Prog. Oceanogr., $77,1-29$.

Lorensen, W. E., \& Cline, H. E. (1987). Marching cubes: A high resolu630 tion 3d surface construction algorithm. SIGGRAPH Comput. Graph., 21,

1 163-169. URL: http://doi.acm.org/10.1145/37402.37422 doi 10.1145/ 37402.37422 . 
Marshall, J., Adcroft, A., Hill, C., Perelman, L., \& Heisey, C. (1997). A finitevolume, incompressible navier stokes model for studies of the ocean on parallel computers. J. Geophys. Res.: Oceans, 102, 5753-5766.

Maxey, M. R., \& Riley, J. J. (1983). Equation of motion for a small rigid sphere in a nonuniform flow. Phys. Fluids, 26, 883-889. doi:10.1063/1.864230

McWilliams, J. C. (1984). The emergence of isolated coherent vortices in turbulent flow. J. Fluid Mech., 146, 21-43.

Murray, R. J. (1996). Explicit generation of orthogonal grids for ocean models. J. Comput. Phys., 126, 251-273.

Okubo, A. (1970). Horizontal dispersion of floatable particles in the vicinity of velocity singularities such as convergences. In Deep sea research and oceanographic abstracts (pp. 445-454). Elsevier volume 17.

Petersen, M. R., Williams, S. J., Maltrud, M. E., Hecht, M. W., \& Hamann, B. (2013). A three-dimensional eddy census of a high-resolution global ocean simulation. J. Geophys. Res.: Oceans, 118, 1759-1774.

Raj, R., Johannessen, J., Eldevik, T., Nilsen, J., \& Halo, I. (2016). Quantifying mesoscale eddies in the lofoten basin. J. Geophys. Res.: Oceans, 121, 45034521.

Saba, V. S., Griffies, S. M., Anderson, W. G., Winton, M., Alexander, M. A., Delworth, T. L., Hare, J. A., Harrison, M. J., Rosati, A., Vecchi, G. A. et al. (2016). Enhanced warming of the northwest atlantic ocean under climate change. J. Geophys. Res.: Oceans, 121, 118-132.

${ }_{655}$ Van der Walt, S., Schönberger, J. L., Nunez-Iglesias, J., Boulogne, F., Warner, J. D., Yager, N., Gouillart, E., \& Yu, T. (2014). scikit-image: image processing in python. PeerJ, 2, e453.

Wang, Y., Beron-Vera, F., \& Olascoaga, M. (2016). The life cycle of a coherent lagrangian agulhas ring. J. Geophys. Res.: Oceans, 121, 3944-3954. 
${ }_{660}$ Weiss, J. (1991). The dynamics of enstrophy transfer in two-dimensional hydrodynamics. Physica D, 48, 273-294.

Winton, M., Anderson, W. G., Delworth, T. L., Griffies, S. M., Hurlin, W. J., \& Rosati, A. (2014). Has coarse ocean resolution biased simulations of transient climate sensitivity? Geophys. Res. Lett., 41, 8522-8529.

Zhang, Y., Liu, Z., Zhao, Y., Wang, W., Li, J., \& Xu, J. (2014a). Mesoscale eddies transport deep-sea sediments. Sci. Rep., 4 .

Zhang, Z., Wang, W., \& Qiu, B. (2014b). Oceanic mass transport by mesoscale eddies. Science, 345, 322-324. 